\title{
Uma arquitetura de referência para sistemas de casas inteligentes de apoio ao cuidado à saúde da perspectiva de sistemas-de-sistemas
}

\author{
Lina Garcés $^{1,2}$, Flavio Oquendo ${ }^{2}$, Elisa Yumi Nakagawa ${ }^{1}$ \\ ${ }^{1}$ ICMC-Universidade de São Paulo (USP) \\ Av. Trabalhador São-carlense, 400 - Centro, 13566-590, São Carlos, SP, Brasil. \\ ${ }^{2}$ IRISA - Université de Bretagne-Sud (UBS) \\ Rue Yves Mainguy, 56000, Vannes, France. \\ linamgr@icmc.usp.br, flavio.oquendo@irisa.fr, elisa@icmc.usp.br
}

\begin{abstract}
Resumo. Sistemas de casas inteligentes para o cuidado da saúde (em inglês Healthcare Supportive Home ou HSH) podem prover serviços de saúde nas residências de pacientes diagnosticados com uma ou múltiplas doenças crônicas, visando principalmente a melhoria da qualidade de vida e da autonomia, bem como a diminuição dos custos dos sistemas públicos de saúde. Contudo, sistemas HSH apresentam grandes desafios principalmente no tocante ao projeto de sua arquitetura e que precisam ser de fato tratados visando sua alta qualidade e viabilidade econômica. Além disso, sistemas de HSH são na maioria dos casos constituídos por diversos outros sistemas que são complexos, independentes, distribuídos e heterogêneos, como por exemplo dispositivos médicos, sistemas de saúde eletrônica (e-Health), sistemas de informação em saúde, entre outros. Dessa forma, eles não devem ser tratados como sistemas monolíticos (como atualmente os são), mas sim como Sistemas-de-Sistemas (do inglês, Systems-of-Systems ou SoS). Nesse cenário, o principal objetivo desta tese foi contribuir para o projeto arquitetural de $\mathrm{HSH}-\mathrm{SoS}^{1}$; para isso, foi estabelecida a HomecARe, uma arquitetura de referência que dá suporte ao reúso sistemático do conhecimento técnico e de domínio, facilitando o projeto e desenvolvimento de HSH-SoS. Para estabelecer a HomecARe, foi adotado um processo sistemático que apoia a engenharia de arquiteturas de referência. Visando analisar a viabilidade e relevância da HomecARe, foi conduzido um amplo estudo de caso no qual a HomecARe foi utilizada para projetar o DiaManT@Home, um HSH-SoS de apoio a pacientes com diabetes mellitus para a autogestão de sua doença. Resultados obtidos evidenciaram a relevância e viabilidade da HomecARe possibilitando construir HSH-SoS reusáveis, interoperáveis, confiáveis, seguros e adaptativos, sendo inclusive uma contribuição inédita para a área de saúde eletrônica, e trazendo avanços nas áreas de arquitetura de referência e $S o S$.
\end{abstract}

Palavras-chaves: Arquitetura de referência, assistência domiciliar, sistemas-desistemas, arquitetura de software, e-Health.

\section{Introdução}

Atualmente, sistemas de HSH têm de fato crescido em importância, dando suporte às atividades diárias de pacientes diagnosticados com doenças crônicas, facilitando sua vida, promovendo sua independência e mantendo-os ativos em suas residências ao invés de permanecerem em hospitais ou clínicas [Bal et al. 2011]. Esse fato também beneficia o serviço público de saúde em função da economia gerada ao manter os pacientes em suas residências

\footnotetext{
${ }^{1}$ Acrônimo de Healthcare Supportive Systems-of-Systems, que será utilizado no restante deste artigo.
} 
[Wartena et al. 2010]. Na última década, a quantidade de sistemas de HSH tem crescido exponencialmente ${ }^{2}$ com foco em diversas doenças, tais como a doença pulmonar obstrutiva crônica, insuficiência renal, AVC, falha cardíaca e doenças neurodegenerativas (Alzheimer e Parkinson). Embora haja uma grande oferta de soluções de HSH, elas ainda apresentam grandes desafios [Maeder and Williams 2017, Majumder et al. 2017]: (i) esses sistemas são na sua grande maioria soluções proprietárias, além de serem monolíticas, altamente acopladas e custosas para os sistemas públicos de saúde; (ii) a maioria desses sistemas não interoperam entre si ou com outros sistemas externos, distribuídos e gerenciados por diferentes entidades, como por exemplo os sistemas de prontuario eletrônico (Electronic Health Record ou EHR, também Personal Health Record ou PHR), sistemas de emergência (SAMU), sistemas de regulação e outros sistemas de informação de saúde (Health Information Systems ou HIS); (iii) sistemas de HSH não apresentam mecanismos para interoperar com sistemas que operem nas casas dos pacientes, como os sistemas domóticos, robôs de assistência/companhia, sistemas de monitoramento de quedas, etc; (iv) as soluções apresentadas pelos sistemas de HSH fornecem uma visão limitada do estado da saúde do paciente, uma vez que foram projetados para doenças específicas sem considerar as particularidades de cada paciente; e (v) sistemas de HSH não possibilitam sua configuração para legislações locais, regionais ou nacionais, bem como para diferentes planos de saúde e demais tecnologias que os pacientes possam possuir em suas residências. Como uma consequência, não há a possibilidade de reusar esses sistemas em contextos diferentes daqueles para os quais foram criados inicialmente.

Por meio de uma revisão sistemática da literatura conduzidas para investigar a existência de diretrizes para o desenvolvimento de sistemas de e-Health e de ambientes assistidos [Garcés et al. 2020, Garcés et al. 2015], foram encontradas 18 arquiteturas de referência (AR) para o domínio de saúde, sendo que dois bons exemplos são o UniversAAL ${ }^{3}$ e a Continua [Wartena et al. 2010]. Uma AR refere-se a uma arquitetura de software que abrange o conhecimento sobre como projetar arquiteturas de software de sistemas de um dado domínio de aplicação [Nakagawa et al. 2014b]. Entre os benefícios de adotar AR temse [Nakagawa et al. 2014b, Martínez-Fernández et al. 2013]: (i) o impacto positivo na produtividade da equipe de desenvolvimento, já que o conhecimento arquitetural é compartilhado e reusado; (ii) a criação de soluções padronizadas e de qualidade para os sistemas de software do domínio; e (iii) aprimoramento da interoperabilidade e capacidade de evolução desses sistemas. Apesar dessas vantagens, nenhuma das 18 AR tratam sistemas de suporte à auto-gestão de múltiplas doenças crônicas ou que possam dar assistência aos pacientes em situações de emergência [Garcés 2018]. Além disso, a interoperabilidade entre os HSH-SoS com sistemas externos e internos às residencias dos pacientes não tem sido o foco dessas AR. Finalmente, essas 18 AR são muito abstratas, requerendo uma curva de aprendizado alta antes de serem utilizadas em projetos de HSH-SoS [Garcés 2018].

O objetivo principal desta tese foi estabelecer a HomecARe, uma AR que dá suporte à construção de HSH-SoS visando diferentes aspectos, em particular, a qualidade, interoperabilidade, padronização, adaptação às necessidades especificas de cada paciente, evolução contínua, baixo acoplamento e reusabilidade, para que esses sistemas de fato assistam ao monitoramento contínuo dos pacientes crônicos em suas residências. Dessa forma, a Home$c A R e$ trouxe importantes avanços no estado da arte nas áreas de ambientes assistidos e HSH-

\footnotetext{
${ }^{2}$ Esses sistemas podem ser consultados nas bases da Comissão Europeia (http://ec.europa.eu/ research/health/index. cfm?pg=projects) e no projeto AAL Joint Programme (http://www . aal-europe.eu/).

${ }^{3}$ http://universaal.sintef9013.com/index.php/en/
} 
SoS. O restante deste artigo é organizado como segue. A Seção 2 descreve os métodos utilizados e os resultados obtidos durante a construção da HomecARe. Os resultados da avaliação dessa AR são apresentados na Seção 3. Finalmente, as considerações mais importantes são discutidas na Seção 4.

\section{Resultados}

Para a construção da HomecARe, foi adotado o ProSA-RA [Nakagawa et al. 2014a], um processo sistemático de quatro etapas para a construção e avaliação de AR: (i) análise do domínio para o qual a AR será concebida; (ii) análise arquitetural; (iii) síntese arquitetural; e (iv) avaliação arquitetural. Resultados das três primeiras etapas são descritos a seguir nesta seção.

\subsection{Análise do Domínio de HomecARe}

Nesta etapa, investigou-se o domínio de atenção domiciliar, incluindo-se os planos de atenção domiciliar de diferentes países, bem como as diretrizes para executar as ações de promoção à saúde, prevenção e tratamento de doenças e reabilitação que possibilita a continuidade do cuidado dos pacientes em seus domicílio. Foram realizados cursos online, e teve-se a assessoria de enfermeiras especialistas no cuidado de pacientes crônicos. Baseado nesse entendimento, foram identificados os objetivos da atenção domiciliar, os processos necessários para atingir tais objetivos, e todos os stakeholders (interessados) envolvidos nesses processos. A seguir, com a finalidade de entender como os sistemas existentes de HSH foram projetados, bem como identificar partes comuns que poderiam ser reutilizadas em sistemas desse domínio, 22 sistemas de HSH foram minuciosamente analisados. Assim, as partes constituintes, funcionalidades e decisões de projeto de cada sistema foram identificadas. Como resultado importante, foi realizada uma analise de qualidade desses sistemas [Garcés et al. 2017] e também estabelecido um modelo de qualidade [Garcés et al. 2016].

\subsection{Análise Arquitetural de HomecARe}

Baseados no conhecimento adquirido na etapa anterior, o domínio para os HSH-SoS foi modelado seguindo o processo definido em [Garcés and Nakagawa 2017], o que resultou em seis modelos complementares, como apresentados na Figura 1: a) modelo de missões representa os objetivos que os HSH-SoS devem cumprir; b) modelo de responsabilidades define os tipos de sistemas constituintes que são requeridos para a execução das missões; c) modelo de capacidades operacionais define as capacidades (ou funcionalidades) requeridas em cada constituinte; d) modelo de dados representa os dados fornecidos por cada constituinte e gerados durante a execução das missões; e) modelo de comunicação descreve as interações entre os constituintes e o HSH-SoS requeridas para a execução das missões; e f) modelo de comportamentos emergentes especifica os possíveis comportamentos dos $\mathrm{HSH}-\mathrm{SoS}$ que podem surgir em tempo de execução. A seguir, foram definidos os requisitos de qualidade mais relevantes para as arquiteturas dos HSH-SoS e que correspondem à capacidade dos HSH-SoS de adaptar-se em tempo de execução, além da interoperabilidade semântica, sintática, técnica e organizacional, bem como a segurança e confiabilidade dos dados e operações.

\subsection{Síntese Arquitetural de HomecARe}

Nessa etapa foram definidas as configurações arquiteturais dos HSH-SoS para atingir as missões, além dos requisitos de qualidade definidos na etapa anterior. Selecionou-se então os padrões arquiteturais mais adequados para os HSH-SoS, sendo eles a SOA (Service-Oriented 

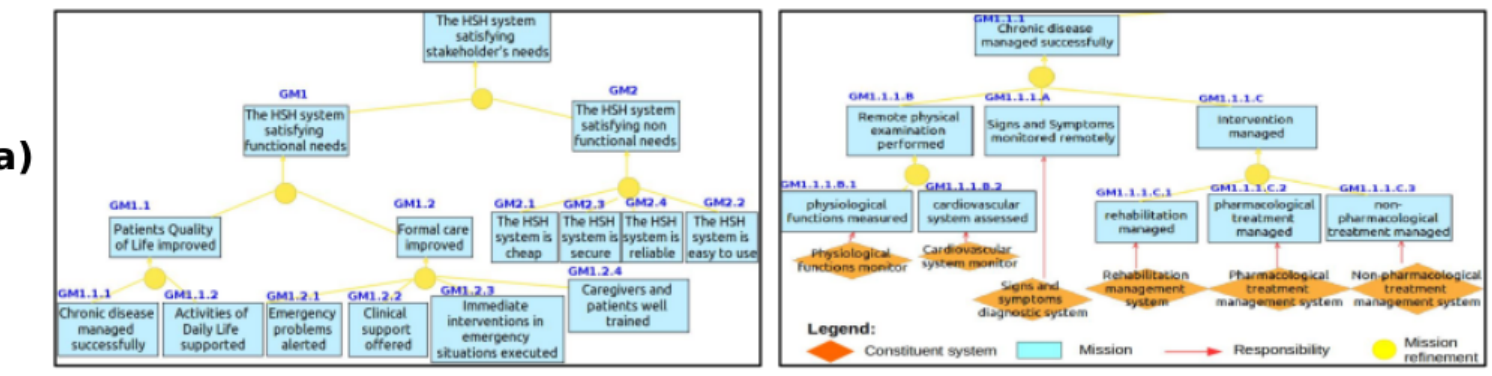

b)
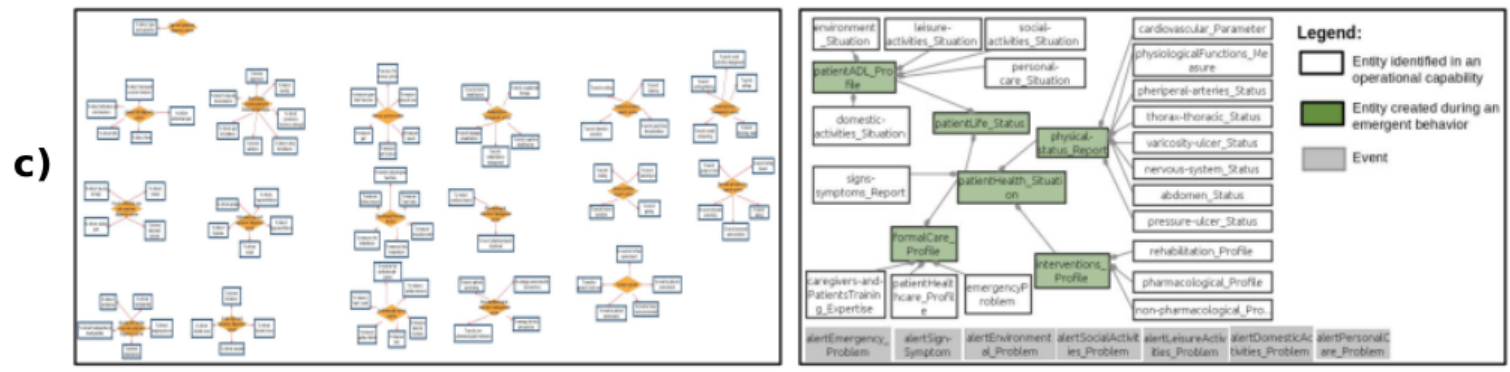

d)
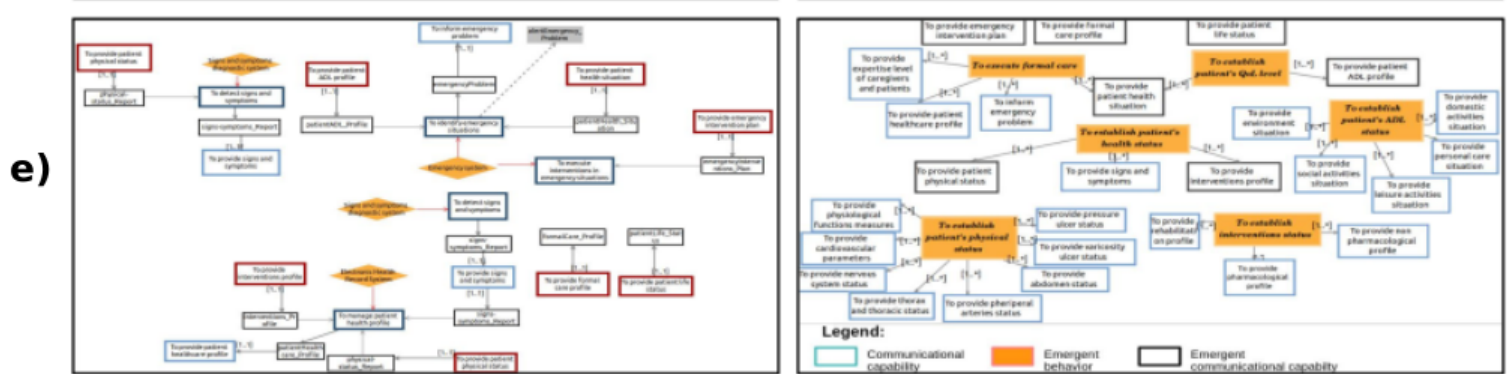

Figura 1. Modelos do domínio em HomecARe.

Architecture), arquitetura reflexiva, MAPE-K (conhecido também como autonomic manager), Publish-Subscribe e o ESB (Enterprise Service Bus). Em conjunto, esses padrões possibilitam a construção de sistemas com características de adaptação, interoperabilidade técnica e organizacional, flexibilidade e evolução contínua. Para abordar os requisitos de segurança, confiabilidade e interoperabilidade semântica e sintática, foram incorporadas à HomecARe as táticas arquiteturais de segurança ponto-a-ponto, não repúdio, mecanismos de tolerância a falhas e brokers. Além disso, para facilitar o entendimento da HomecARe, diferentes visões foram construídas, sendo que a visão geral é mostrada na Figura 2. Essa visão define todos os elementos de software (serviços, repositórios, modelos) que compõem a HomecARe e que foram organizadas em camadas. Para atingir cada missão definida no modelo de missões (Figura 1.a), foi definida uma coleção de serviços que interagem para executar os processos alinhados com a missão. Isso foi representado usando outros seis diferentes tipos de diagramas arquiteturais: (i) diagrama de processos vinculados à missão; (ii) diagrama de capacidades dos serviços fornecidos pelos constituintes envolvidos nos processos; (iii) diagrama das interfaces desses serviços; (iv) diagrama da arquitetura dos serviços; e (v) diagrama dos constituintes participantes. Um total de 129 diagramas compõem a HomecARe, quantidade essa que se faz necessária para de fato descrever completamente uma AR.

\section{Avaliação de HomecARe}

Para avaliar a viabilidade e relevância da HomecARe, um estudo de caso exploratório foi planejado e conduzido seguindo diretrizes sistemáticas propostas em [Runeson et al. 2012], bem como o envolvimento de pessoas externas aos interesses desta tese visando minimizar as ameaças à validade do estudo. Nesse estudo de caso, a arquitetura da DiaManT@Home, 


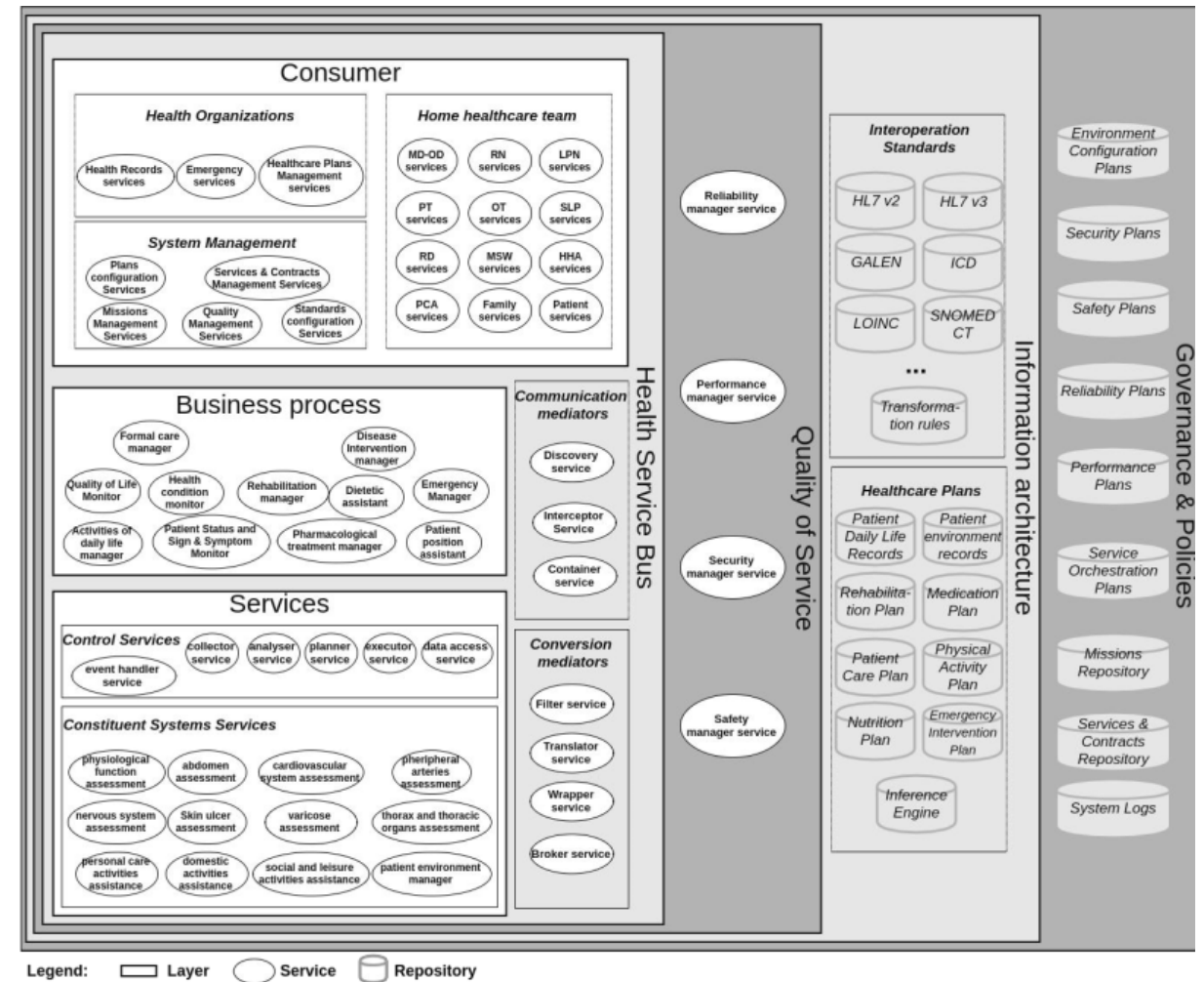

Figura 2. Visões em camadas de HomecARe.

um HSH-SoS de assistência domiciliar para pacientes diagnosticados com diabetes mellitus, foi projetada utilizando-se a HomecARe. Duas métricas foram utilizadas: tempo e esforço gasto para a construção da arquitetura do DiaManT@Home. Como resultado, observou-se que utilizando-se a HomecARe, o tempo para criar a arquitetura foi entre 52\% e 58\% menor do que o gasto em projetos similares, requerendo inclusive uma quantidade similar de esforço dos arquitetos. Isso evidencia um alto reuso de conhecimento por meio da HomecARe, e no caso do DiaManT@Home, 92\% dos elementos necessários para atingir seus requisitos foram reusados de HomecARe. Adicionalmente, a arquitetura de DiaManT@Home foi avaliada com relação aos requisitos de qualidade, a saber interoperabilidade (em todos seus níveis), adaptação em tempo de execução, segurança, confiabilidade e flexibilidade. Tal avaliação foi realizada por meio do uso de cenários de qualidade, uma técnica largamente recomendada para avaliações empíricas na área de arquitetura de software. Resultados positivos foram atingidos para todos esses requisitos de qualidade.

\section{Considerações Finais}

Essa tese trouxe importantes contribuições em diferentes perspectivas tanto na abrangência quanto em profundidade, sendo elas [Garcés 2018]: (i) análise de qualidade de sistemas de software no domínio de ambientes assistidos e e-Health [Garcés et al. 2017, Garcés et al. 2016] como são os HSH-SoS; (ii) apoio aos arquitetos e desenvolvedores no entendimento do estado da arte em AR para as áreas de saúde e ambientes assistidos [Garcés et al. 2015, Garcés et al. 2020]; (iii) HomecARe, uma arquitetura de referência para HSH-SoS para o projeto desses sistemas visando sua qualidade em termos de interoperabilidade, adaptação em tempo de execução, segurança, confiabilidade, flexibilidade e reúso; (iv) projeto arquitetural de DiaManT@Home, um sistema de atenção domiciliar para pacientes diagnosticados com diabetes mellitus [Garcés et al. 2019]; e (v) artefatos para a 
construção de AR para SoS em outros domínios de aplicação [Garcés and Nakagawa 2017, Garcés et al. 2018]. A maioria desses resultados foram já publicados em eventos nacionais e internacionais, bem como em revistas de alto impacto nas áreas de ciências da computação e computação aplicada à saúde.

Além disso, trabalho no sentido de disseminar a HomecARe para que seja utilizada em projetos de HSH-SoS no Brasil e no exterior está sendo realizado. Adicionalmente, a experiência de desenvolver HomecARe, como AR para SoS na área de saúde está sendo utilizada para apoiar outros projetos de pós-graduação conduzidos no START, grupo de pesquisa em arquitetura de software do ICMC-USP. Finalmente, esta tese de doutorado esteve vinculada ao convênio acadêmico internacional para dupla titulação de doutorado assinado entre o ICMC-USP e a Université de Bretagne Sud, França.

Agradecimentos: Os autores agradecem à FAPESP (Processos No.: 2013/20317-9, 2015/19192-2, 2017/06195-9, 2018/07437-9) e à CAPES.

\section{Referências}

Bal, M., Shen, W., Hao, Q., and Xue, H. (2011). Collaborative smart home technologies for senior independent living: A review. In 15th International Conference on Computer Supported Cooperative Work in Design (CSCWD), pages 481-488, Lausanne, Switzerland. IEEE.

Garcés, L., Ampatzoglou, A., Avgeriou, P., and Nakagawa, E. Y. (2015). A comparative analysis of reference architectures for healthcare in the ambient assisted living domain. In 2015 IEEE 28th International Symposium on Computer-Based Medical Systems, pages 270-275, Sao Carlos, SP, Brazil. IEEE.

Garcés, L., Ampatzoglou, A., Avgeriou, P., and Nakagawa, E. Y. (2017). Quality attributes and quality models for ambient assisted living software systems: A systematic mapping. Information and Software Technology, 82(Supplement C):121 - 138.

Garcés, L. and Nakagawa, E. Y. (2017). A process to establish, model and validate missions of systems-of-systems in reference architectures. In Proceedings of the Symposium on Applied Computing, SAC '17, pages 1765-1772, New York, NY, USA. ACM.

Garcés, L., Oquendo, F., and Nakagawa, E. (2020). Assessment of reference architectures and reference models for ambient assisted living systems: Results of a systematic literature review. International Journal of E-Health and Medical Communications (IJEHMC), pages $1-31$.

Garcés, L., Oquendo, F., and Nakagawa, E. Y. (2016). A quality model for aal software systems. In 2016 IEEE 29th International Symposium on Computer-Based Medical Systems (CBMS), pages 175-180, Belfast, Nothern Ireland, UK. IEEE.

Garcés, L., Oquendo, F., and Nakagawa, E. Y. (2018). Towards a taxonomy of software mediators for systems-of-systems. In Proceedings of the VII Brazilian Symposium on Software Components, Architectures, and Reuse, SBCARS '18, pages 53-62, New York, NY, USA. ACM.

Garcés, L., Vicente, I. Z., and Nakagawa, E. Y. (2019). Software architecture of a health care supportive home system to assist patients with diabetes mellitus. In 2019 IEEE 32th International Symposium on Computer-Based Medical Systems, pages 1-4, Cordoba, Spain. IEEE.

Garcés, L. (2018). A reference architecture for healthcare supportive home systems from a systems-of-systems perspective. phdthesis, Institute of Mathematics and Computer Sciences - ICMC at University of Sao Paulo-USP, and Institut de recherche en informatique et systèmes aléatoires - IRISA at Université de Bretagne-Sud - UBS.

Maeder, A. and Williams, P. (2017). Precision Healthcare through Informatics, volume MEDINFO 2017 of Studies in Health Technology and Informatics, chapter Health Smart Homes: New Challenges, pages 166-169. 2017 International Medical Informatics Assiciation (IMIA) and IOS Press, Xiamen, China.

Majumder, S., Aghayi, E., Noferesti, M., Memarzadeh-Tehran, H., Mondal, T., Pang, Z., and Deen, M. (2017). Smart homes for elderly healthcare-recent advances and research challenges. Sensors (Basel)., 17(11, 2496):2-32.

Martínez-Fernández, S., Ayala, C., Franch, X., and Marques, H. M. (2013). Benefits and Drawbacks of Reference Architectures. In Drira, K., editor, 7th European Conference on Software Architecture (ECSA)., pages 307-310, Montpellier, France. Springer Berlin Heidelberg.

Nakagawa, E. Y., Guessi, M., Maldonado, J. C., Feitosa, D., and Oquendo, F. (2014a). Consolidating a Process for the Design, Representation, and Evaluation of Reference Architectures. In 10th Working IEEE/IFIP Conference on Software Architecture (WICSA), pages 143-152, Sydney, Australia. IEEE/IFIP.

Nakagawa, E. Y., Oquendo, F., and Maldonado, J. C. (2014b). Reference Architectures. In Oussalah, M. O., editor, Software Architecture: Principles, Techniques, and Tools, chapter 2, pages 55--82. Wiley, London, 1st edition.

Runeson, P., Host, M., Rainer, A., and Regnell, B. (2012). Case Study Research in Software Engineering: Guidelines and Examples. Wiley Publishing, USA, first edition.

Wartena, F., Muskens, J., Schmitt, L., and Petkovic, M. (2010). Continua: The reference architecture of a personal telehealth ecosystem. In 12th IEEE International Conference on e-Health Networking, Applications and Services (Healthcom), Lyon, France. IEEE. 